\title{
Incidence, Prevalence, and Outcomes of Pediatric Trauma in Rural Appalachia (West Virginia) From 2017 to 2019
}

Pavithra Ellison ${ }^{1}$, Daniel Cifarelli ${ }^{2}$, Alexandra Pearce ${ }^{3}$, Lucas Moore $^{4}$, Dan Parrish ${ }^{5}$, Matthew Ellison ${ }^{1}$, Alyssa Fazi ${ }^{1}$, Trey Vanek ${ }^{1}$, Hal Meltzer ${ }^{2}$, Jennifer Knight ${ }^{6}$

1. Anesthesiology, West Virginia University School of Medicine, Morgantown, USA 2. Neurosurgery, West Virginia University School of Medicine, Morgantown, USA 3. Medicine, West Virginia University School of Medicine, Morgantown, USA 4. Biostatistics and Epidemiology, West Virginia University School of Medicine, Morgantown, USA 5. Surgery, West Virginia University School of Medicine, Morgantown, USA 6. Surgical Trauma and Critical Care, West Virginia University School of Medicine, Morgantown, USA

Corresponding author: Pavithra Ellison, pavithra.ellison@wvumedicine.org

\section{Abstract}

\section{Background}

Appalachian rural pediatric trauma has its unique incidence, presentation, and distribution due to the mechanisms of injury, geographic location, access to care, and social issues.

\section{Purpose}

To review, analyze, and understand pediatric trauma in West Virginia during the period 2017-2019.

\section{Materials and methods}

After institutional review board approval, the statewide trauma database was queried and analyzed in a retrospective cohort study for all pediatric trauma ages zero to 18 from 2017-2019 in the Appalachian regions one through four in West Virginia.

The following were analyzed: gender, injury mechanism, Glasgow Coma Scale Score (GCS) at admission, injury severity score (ISS), toxicology screen results, hospital length of stay, duration of ventilatory support, number of procedures performed during admission, presence of non-accidental trauma, cardiac arrest, patient discharge disposition, and mortality.

\section{Results}

One-thousand eighty-two (1182) patients between the ages of zero to 18 were admitted to the trauma center. An average of $37 \%$ was female and $63 \%$ male. In the $11-18$ age group, $24 \%$ were female and $76 \%$ were

Review began 03/21/2021 Review ended 03/29/2021 Published 04/01/2021

(๑) Copyright 2021 Ellison et al. This is an open access article distributed under the terms of the Creative Commons Attribution License CC-BY 4.0., which permits unrestricted use, distribution, and reproduction in any medium, provided the original author and source are credited. male. Most injuries were due to blunt force (89\%), followed by penetrating injuries (7.2\%) and burns (1.4\%). The majority had minor or moderate injuries with 95\% receiving a Glasgow Coma Scale (GCS) >13 and $72 \%$ listed as minor on the injury severity score (ISS). Children in ages 0 - 2 years had the highest proportion of poor (0-8) GCS scores, high ISS (>14) scores, most hospital admission days, most days on a ventilator, highest mortality, most pre-hospital cardiac arrests, child abuse, burns, and placement with child protective services. An average of $31 \%$ of children tested, and $17 \%$ in the age group of $0-2$ had a positive toxicology screen. There were 3670 procedures done in total and the most common procedure performed was an ultrasound of the abdomen. Procedures were performed in $90 \%$ of the patients.

\section{Conclusions and relevance}

Based on our study, the zero to two-year-old pediatric trauma patients are most vulnerable to poor outcomes and may need targeted preventative interventions. Toxicology screens may need to be more widely implemented in pediatric trauma in the Appalachian region.

Rural trauma in Appalachia has endemic issues related to substance abuse, poverty, and a lower degree of social support as compared to urban areas. Although the distribution of injury may follow a national distribution, mechanism, management, and outcomes can vary.

Categories: Pediatrics, Epidemiology/Public Health, Trauma

Keywords: trauma pediatric, child abuse, toxicology screen, patient outcomes

\section{Introduction}

For the past three decades, there has been an evolving trend towards regional centers of excellence for specialty care in pediatric cancer, cardiovascular medicine, and solid organ transplantation [1-4]. However, 
the ability of patients and their families to choose a specialty center is unfeasible for urgent traumatic injury, leaving local pediatric trauma designated facilities as the first and only option for management [5]. Maintaining practice standards with a routine review of patient outcomes in regional trauma centers provides metrics for outcomes improvement.

Rural Appalachian pediatric trauma centers encounter patients with unique injury mechanisms and whose demographics are dissimilar from those in urban centers. Therefore, outcomes data from urban or suburban centers may or may not be relevant in the rural setting [6]. In this retrospective cohort study, we reviewed the admission and disposition data of pediatric trauma patients at our institution (the largest pediatric trauma center in the state) over a three-year period. This facility serves as the primary referral center for a rural population, with a density of approximately 77 people per square mile. When compared to the adjacent states of Pennsylvania and Ohio, with 286 people per square mile, the data reviewed in this study could provide a better means of providing optimal care and, ultimately, injury prevention in a rural population.

\section{Materials And Methods \\ Methods}

We conducted a retrospective cohort study in which we aimed to describe the incidence or prevalence of different pediatric trauma in rural Appalachia and describe its association with variables of interest, including gender, injury mechanism, Glasgow Coma Scale Score (GCS) at admission, injury severity score (ISS), drug test results, hospital length of stay, duration of ventilatory support, number of procedures performed during admission, presence of non-accidental trauma, cardiac arrest, patient discharge disposition, and mortality. After approval from our institutional review board (IRB), the admission and outcomes database maintained by our health system was queried for all admissions between 2017 and 2019. The primary inclusion criterion was an age of less than 18 years at the time of presentation.

\section{Statistics}

Statistical analysis was performed using the Statistical Package for the Social Sciences (SPSS) software v. 26 (IBM Corporation, Armonk, NY). The data were stratified based on age, with primary groupings of zero to two years, three to five years, six to 10 years, and 11-18 years, and used for the determination of mean, percentages, and interquartile range. The minimum variables obtained included gender, injury mechanism, GCS at admission, ISS, toxicology screen results, hospital length of stay, duration of ventilatory support, number of procedures performed during admission, presence of non-accidental trauma, cardiac arrest, patient discharge disposition, and mortality.

\section{Results}

\section{Patient demographics}

During the period of 2017-2019, a total of 1,182 pediatric trauma patients were admitted to health system facilities for treatment. Of these, 739 (63\%) were identified as male. As patient age increased, there was a predominance of trauma in males, reaching a maximum of $76 \%$ male patients in the 11 to 18 -year-old patient cohort (summarized in Table 1).

\begin{tabular}{|c|c|c|c|}
\hline Age (years) & Female n (\%) & Male n (\%) & Unknown n (\%) \\
\hline $0-2$ & 125 (44.17) & 158 (55.83) & $0(0.00)$ \\
\hline $3-5$ & 108 (44.63) & 133 (54.96) & $1(0.41)$ \\
\hline $6-10$ & 136 (38.64) & $216(61.36)$ & $0(0.00)$ \\
\hline $11-18$ & $72(23.61)$ & 232 (76.07) & $1(0.33)$ \\
\hline Total & 441 (37.31) & 739 (62.52) & $2(0.17)$ \\
\hline
\end{tabular}

TABLE 1: Pediatric trauma categorization by gender

\section{Regional pediatric trauma distribution}

Based on districts identified by the West Virginia Department of Health and Human Resources Bureau for Children and Families, the state is divided into four regions, Regions 1-4.

Most patients in this study were from Regions 1 and 3 (n=1028). Sixty-nine percent or 581 out of 842 patients from Region 1 were directly admitted to the largest tertiary institution. In this study, 101 patients were referred from facilities outside of West Virginia, including 72 from Maryland, eight from Ohio, 19 from Pennsylvania, and two from Virginia (Table 2). 


\section{Cureus}

\begin{tabular}{|c|c|c|c|c|}
\hline Age (years) & Region 1 n (\%) & Region 2 n (\%) & Region 3 n (\%) & Region 4 n (\%) \\
\hline $0-2$ & 194 (76.68) & $3(1.19)$ & 46 (18.18) & $10(3.95)$ \\
\hline $3-5$ & $181(81.90)$ & $2(0.90)$ & $34(15.38)$ & $4(1.81)$ \\
\hline $6-10$ & 252 (77.06) & $4(1.22)$ & 59 (18.04) & $12(3.67)$ \\
\hline $11-18$ & 215 (76.79) & $4(1.43)$ & 47 (16.79) & $14(5.00)$ \\
\hline Total & $842(77.89)$ & $13(1.20)$ & $186(17.21)$ & $40(3.70)$ \\
\hline
\end{tabular}

TABLE 2: Pediatric trauma categorization by age and region

\section{Presentation of pediatric trauma injuries}

Patients were classified into the three major injury categories, which include blunt trauma (including motor vehicle collision), burns, and penetrating injury (including gunshot wounds). Most injuries were caused by blunt trauma (89\% across all age groups), with burns and penetrating injuries comprising $1.4 \%$ and $7.2 \%$, respectively. A trend towards a higher incidence of burns, relative to other injury mechanisms, was seen in patients aged zero to two years ( $76 \%$ ), as compared to $7 \%-11 \%$ in all other age cohorts, while the incidence of penetrating injuries was evenly distributing based on age (Table 3).

\begin{tabular}{|c|c|c|c|c|c|}
\hline Age (years) & Blunt n (\%) & Burn n (\%) & Penetrating n (\%) & Other $\mathbf{n}(\%)$ & N/A n (\%) \\
\hline $0-2$ & $243(85.87)$ & $13(4.59)$ & $19(6.71)$ & $7(2.47)$ & $1(0.35)$ \\
\hline $3-5$ & 216 (89.26) & $1(0.41)$ & $16(6.61)$ & $6(2.48)$ & $3(1.24)$ \\
\hline $6-10$ & 316 (89.77) & $1(0.28)$ & $28(7.95)$ & $3(0.85)$ & $4(1.14)$ \\
\hline $11-18$ & $277(90.82)$ & $2(0.66)$ & $23(7.54)$ & $1(0.33)$ & $2(0.66)$ \\
\hline Total & $1052(89.00)$ & $17(1.44)$ & $86(7.28)$ & $17(1.44)$ & $10(0.85)$ \\
\hline
\end{tabular}

TABLE 3: Pediatric trauma categorization by mechanism of injury

N/A: not documented

\section{Level of consciousness and injury severity at admission}

Most patients had minor or moderate injuries based on GCS and ISS (95\% had GCS > 13 and 72\% were listed as minor on the ISS). For severe injuries, patients in the zero to two-year age group had the highest incidence of poor GCS and ISS scores (53\% of patients with GCS $\leqslant 8$ and $56 \%$ of patients designated as severe on the ISS (\$48) fell in this age group (summarized in Tables 4-5).

\begin{tabular}{|c|c|c|c|c|}
\hline Age (years) & Severe (0-8) n (\%) & Moderate (9-12) n (\%) & Mild (13-15) n (\%) & Unknown n (\%) \\
\hline $0-2$ & $17(6.01)$ & $5(1.77)$ & $260(91.87)$ & $1(0.35)$ \\
\hline $3-5$ & $5(2.07)$ & $4(1.65)$ & $233(96.28)$ & $0(0.00)$ \\
\hline $6-10$ & $6(1.70)$ & $7(1.99)$ & $339(96.31)$ & $0(0.00)$ \\
\hline $11-18$ & $4(1.31)$ & $3(0.66)$ & 299 (98.03) & $0(0.00)$ \\
\hline Total & $32(2.71)$ & 18(1.52) & 1131 (95.69) & $1(0.08)$ \\
\hline
\end{tabular}

\section{TABLE 4: Pediatric trauma categorization by GCS score at time of admission}




\section{Cureus}

\begin{tabular}{|c|c|c|c|c|c|}
\hline Age (years) & Minor (\%) & Moderate (\%) & Serious (\%) & Severe (\%) & Critical (\%) \\
\hline $0-2$ & 188 (66.43) & 69 (24.38) & $11(3.89)$ & 14 (4.95) & $1(0.35)$ \\
\hline $3-5$ & 179 (73.97) & 56 (23.14) & $3(1.24)$ & 4 (1.65) & $0(0.00)$ \\
\hline $6-10$ & 276 (78.41) & 57 (16.19) & $12(3.41)$ & 7 (1.99) & $0(0.00)$ \\
\hline $11-18$ & 212 (69.51) & 78 (25.57) & 9 (2.95) & 5 (1.64) & $1(0.33)$ \\
\hline Total & 855 (72.34) & $260(22.00)$ & $35(2.96)$ & $30(2.54)$ & $2(0.17)$ \\
\hline
\end{tabular}

TABLE 5: Pediatric trauma categorization by ISS at time of admission

ISS: injury severity score

\section{Toxicology screen}

A total of 275 patients (23\%) underwent urine or serum toxicology screen in the emergency department. Of these, $85(31 \%)$ were found to be positive. There was an increasing percentage of positive toxicology screens with advancing patient age as seen in the following groups (Age 0-2: 18\% (7/39), 3-5: 21\% (12/57), 6-10: 35\% (29/82), 11-18: 37/97 (38\%)). Overall, 7.19\% of children in the study had a positive toxicology screen (summarized in Table 6).

\begin{tabular}{|l|l|l|l|}
\hline Age (years) & $\begin{array}{l}\text { Testing completed } \mathbf{n} \\
(\%)\end{array}$ & $\begin{array}{l}\text { Positive } \mathbf{n} \\
(\%)\end{array}$ & No Testing $\mathbf{n}(\%)$ \\
\hline $0-2$ & $39(13.78)$ & $7(17.95)$ & $244(86.22)$ \\
\hline-5 & $57(23.55)$ & $12(21.05)$ & $185(76.45)$ \\
\hline $6-10$ & $82(23.30)$ & $29(35.37)$ & $269(76.42)$ \\
$11-18$ & $97(31.80)$ & $37(38.14)$ & $208(68.20)$ \\
\hline Total & $275(23.27)$ & $85(30.91)$ & $906(76.65)$ \\
\hline
\end{tabular}

National Averages for Alcohol and Drug Use in Pediatric Trauma Cases in 2016 Source: NTDB Pediatric Report 2016. Published online 2016:128

$\begin{array}{lllr}\text { Alcohol use } & \text { Number (\%) } & \text { Drug use } & \text { Number (\%) } \\ \text { No (confirmed by test) } & 25109(17.80) & \text { No (confirmed by test) } & 9661(6.85) \\ \text { No (not tested) } & 88850(62.99) & \text { No (not tested) } & 103184(73.15) \\ \text { Yes (beyond the legal limit) } & 2953(2.09) & \text { Yes (confirmed by test; illegal drug use) } & 6031(4.28) \\ \text { Yes (trace amounts) } & 2066(1.46) & \text { Yes (confirmed by test; prescription drug) } & 3519(2.49) \\ \text { Not Applicable } & 16725(1.86) & \text { Not Applicable } & 10574(7.50) \\ \text { Not Known/Not Recognized } & 5348(3.79) & \text { Not Known/Not Recognized } & 8082(5.73) \\ \text { Total } & 141051(100) & \text { Total } & 141051(100)\end{array}$

\section{TABLE 6: Pediatric trauma toxicology testing and results}

NTDB: National Trauma Data Bank®

Children between the ages of zero to two had the highest proportion of poor GCS scores (0-8). High ISS scores (>14), most hospital admission days, most days on a ventilator, highest mortality, most pre-hospital cardiac arrests, the highest incidence of child abuse, most burns, and highest placement with child protective services.

\section{Hospital course and discharge outcomes}

Patients aged zero to two had the longest length of stay, most days on a ventilator, highest mortality, most 


\section{Cureus}

prehospital cardiac arrests, and the highest incidence of child abuse. The average length of stay for patients in all age groups was $2.61 \pm 4.67$ days (Tables 7-8).

\begin{tabular}{|c|c|c|c|}
\hline Age (years) & Number in age group & Mean (STDEV*) & Minimum, Maximum \\
\hline $0-2$ & 283 & $3.42(8.22)$ & 0,90 \\
\hline $3-5$ & 242 & $2.19(1.60)$ & 0,16 \\
\hline $6-10$ & 352 & 2.34 (2.99) & 0,34 \\
\hline $11-18$ & 305 & $2.48(2.92)$ & 0,37 \\
\hline
\end{tabular}

TABLE 7: Pediatric trauma categorization by age for length of stay in hospital (days)

*STDEV: standard deviation

\begin{tabular}{|c|c|c|c|}
\hline Age (years) & Number & Mean (STDEV*) & Minimum, Maximum \\
\hline $0-2$ & 23 & 10.30 (14.18) & 1,61 \\
\hline $3-5$ & 9 & $2.55(1.24)$ & 1,5 \\
\hline $6-10$ & 11 & 3.54 (3.78) & 1,12 \\
\hline $11-18$ & 8 & $2.62(1.84)$ & 1,7 \\
\hline
\end{tabular}

TABLE 8: Pediatric trauma categorization by age for assisted ventilation (days)

STDEV: standard deviation

Overall, 15 patients (1.27\%) did not survive their injuries. Ten of these belonged to the zero to two age group (3.53\%), one in the three to five age group $(0.41 \%)$, one in the six to 10 age group $(0.28 \%)$, and three in the 11-18-year-old age group (0.98\%). All patients, except those in the zero to two-year-old age group, had less than $1 \%$ mortality (Table 9 ).

\begin{tabular}{|c|c|c|}
\hline Age (years) & Alive $n(\%)$ & Deceased n (\%) \\
\hline $0-2$ & $273(96.47)$ & $10(3.53)$ \\
\hline $3-5$ & 241 (99.59) & $1(0.41)$ \\
\hline $6-10$ & 351 (99.72) & $1(0.28)$ \\
\hline $11-18$ & 302 (99.02) & $3(0.98)$ \\
\hline Iotal & $1167(98.73)$ & $15(1.27)$ \\
\hline
\end{tabular}

TABLE 9: Pediatric trauma categorization by age and condition at discharge

The incidence of prehospital cardiac arrest in all patients was $1.18 \%(\mathrm{n}=14)$. It was less than $1 \%$ in all age groups except those aged zero to two, where it was $3.18 \%(\mathrm{n}=9)$ (Table 10). 


\section{Cureus}

\begin{tabular}{|c|c|c|}
\hline Age (years) & Yes Number (\%) & No Number (\%) \\
\hline $0-2$ & $9(3.18)$ & $274(96.82)$ \\
\hline $3-5$ & $0(0.00)$ & $242(100)$ \\
\hline $6-10$ & $3(0.85)$ & 349 (99.15) \\
\hline $11-18$ & $2(0.66)$ & 303 (99.34) \\
\hline Total & $14(1.18)$ & 1168 (98.82) \\
\hline
\end{tabular}

\section{TABLE 10: Pediatric trauma categorization by age and by pre-hospital cardiac arrest}

Yes: History of pre-hospital cardiac arrest

No: No history of pre-hospital cardiac arrest

Similarly, the overall incidence of child abuse was $1.52 \%(\mathrm{n}=18)$, with the overwhelming majority in the zero to two-year-old age group $(\mathrm{n}=15)$. The age group with the second-highest occurrence was the three to fiveyear-old group $(n=2)$, followed by the six to 10 age category $(n=1)$. There were no reported instances of child abuse in the 11-18 category (Table 11 ).

$\begin{array}{ll}\text { Age (years) } & \text { Number }(\%) \\ 0-2 & 15(5.30) \\ 3-5 & 2(0.83) \\ 6-10 & 1(0.28) \\ 11-18 & 0(0.00) \\ \text { Total } & 18(1.52)\end{array}$

TABLE 11: Pediatric trauma categorization by age and incidence of child abuse

The age group with the highest incidence of child protective services (CPS) involvement or burn center referral was zero to two years at $12 \%$.

There was a total of 3670 procedures performed in all patients, the most common of which was the abdominal ultrasound. Procedures were performed in 1063 (90\%) of all patients. The average number and standard deviation of procedures performed in each patient were approximately $3.4 \pm 3$ (Table 12).

\begin{tabular}{|c|c|c|c|}
\hline Age Category (years) & Number (\%) & Mean (STDEV) & Minimum, Maximum \\
\hline $0-2$ & 224 (79.15) & 3.34 (3.77) & 1,26 \\
\hline $3-5$ & 221 (91.32) & $2.82(2.04)$ & 1,14 \\
\hline $6-10$ & $335(95.17)$ & $3.42(2.82)$ & 1,29 \\
\hline $11-18$ & 283 (92.79) & $4.05(3.34)$ & 1,26 \\
\hline
\end{tabular}

TABLE 12: Pediatric trauma categorization by age and procedures performed

STDEV: standard deviation

The percentage of patients who underwent at least one procedure in each group were: $79 \%$ in the zero to two age group ( $3.34 \pm 3.77$ procedures with a maximum number of 26$) ; 91 \%$ in the three to five-year-old age group $(2.82 \pm 2.04$ procedures with a maximum of 14$)$; $91 \%$ in the six to 10 age group $(3.42 \pm 2.82$ procedures with a maximum of 29$)$; $95 \%$ in the 11 -18-year-old age group (3.42 \pm 2.82 procedures with a maximum of 


\section{Discussion}

Unintentional trauma is the number one cause of death in children and adolescents in the United States [7]. Although pediatric trauma populations can be similarly characterized, there are unique challenges faced in different regions of the country. In this investigation, we analyzed the characteristics of pediatric trauma patients served by the largest tertiary hospital and our state health system. Upon review of the literature, it is evident that there are differences between urban and rural pediatric trauma populations [5-6].

A common theme throughout the literature is that males have a higher propensity for traumatic injury than females, as well as a higher incidence of death due to traumatic injury [8-11]. Our data demonstrated a comparable trend, with $63 \%$ of all analyzed pediatric trauma cases being male. We also found that there was an increase in the proportion of males versus females experiencing traumatic injuries with rising patient age. This likely reflects the increased risk-taking behavior seen in adolescent males relative to other age and gender categories. Accordingly, a similar study in Illinois found that higher age and male gender were strong predictors for a positive toxicology screen among their pediatric trauma cases [12]. Additional data are needed to determine if this is true for our population, but it is important to consider the relationship between drug use, male gender, and age and how it could contribute to the trend seen in our study population.

Drug use in pediatric patients has not only been associated with increasing age and male gender but also with lower socioeconomic status and more violent injury mechanism [13]. In our study population, $23 \%$ of all patients were screened for drug use. Thirty-one percent of these tests were positive, with $38 \%$ of the positive tests in the 11-18-year-old age group. Several other studies have demonstrated a similar proportion of positive drug screens [11-14]. One study demonstrated positive tests in 39\% of the 12 -18-year-old trauma group who met the criteria for drug screening while another revealed a $34 \%$ positive rate in 13 -19-year-old trauma patients [13-14]. However, this is not ubiquitous across the literature. For example, Indiana and Illinois have significantly different incidences of positive screens; $18 \%$ versus $9 \%$ in their adolescent trauma population [10-11]. Based on records from the 2016 National Trauma Data Bank, $4.28 \%$ of pediatric trauma cases tested positive for illegal drugs and $2.09 \%$ tested positive for alcohol content above the legal limit [15]. Although our data does not differentiate between drug and alcohol use, $7.2 \%$ of our pediatric study population had a positive drug test, suggesting that the Appalachian region may have more drug-positive pediatric trauma cases than the national average. Despite variances in literature, the proportion of pediatric trauma cases with positive toxicology screens in our region is in the upper limit of reported data. Further studies to ascertain whether pediatric trauma, its mechanism of injury, associated factors like drug and alcohol association have evolved and changed over the last decade in the Appalachian region. Our goal in the next study is to analyze the evolution of trauma and its management and outcomes over the last decade in the Appalachian region. An in-depth analysis of the subtypes of the toxicology screen, correlating it to injury type (violent versus non-violent injuries, accidental versus intentional injuries), age and sex, and race may give us an opportunity for early preventative intervention. One of the shortcomings of this pilot study was the lack of trauma distribution based on race. Future studies will include race in their analysis.

Although the classification of injury mechanisms may differ in the literature, one common theme is the high prevalence of blunt force trauma-related injuries in pediatric populations [8,10]. Our data follow a similar pattern, with $89 \%$ of all reported injuries secondary to blunt force trauma. It is important to note that motor vehicle accidents are included in this category. The World Health Organization reported that road-related injuries are the leading cause of death in children aged 15-19 and the second leading cause in those aged five to 14 in the United States [16]. Another concerning point is the high incidence (5\%) of child abuse in the zero-to-two-year-old age group that significantly exceeds that seen in any other age group. This clearly demonstrates a need for hypervigilance in an extremely vulnerable group of patients. The zero-to-two-yearold group also had injuries that were more severe, as well as the highest proportion of the following: poor (08) GCS scores, high ISS (>14) scores, longer length of stay, increased ventilator duration, higher mortality, more pre-hospital cardiac arrests, more burns, and higher incidence of placement with CPS. This suggests that our injury prevention strategies may need to specifically target parents with children at or under the age of two.

In the future, as we expand the cohort to the last decade of data, we hope to utilize the information gathered to help guide the development of prevention strategies targeting the most common injuries seen in rural pediatric populations.

\section{Conclusions}

Male patients represented almost 2/3 of pediatric trauma with blunt force injuries being the most predominant mechanism of injury. Zero-to-two-year-old patients were the most vulnerable to poor outcomes and may need targeted preventative interventions. Due to the high incidence of positive toxicology screens, these tests may need to be more widely implemented in pediatric trauma in the Appalachian region. Overall, there are many similarities between the information that we have ascertained from rural pediatric trauma from the Appalachian region and trauma records across the United States. However, there were also many parameters, namely, the high incidence of positive toxicology screens, which 
may uniquely reflect the population we serve. Rural pediatric trauma may have endemic issues related to substance abuse, poverty, and lower degrees of social support when compared to urban areas. Nevertheless, substantial work is needed to delineate specific trends in various parameters like injury mechanisms across age groups. This will be an important aid to the development of prevention strategies to target our most vulnerable population: the children of the Appalachian region.

\section{Additional Information \\ Disclosures}

Human subjects: Consent was obtained or waived by all participants in this study. West Virginia University Office of Research Integrity and Subject Protection issued approval 1911800005. Presentation, evolution and management of Pediatric trauma at WVU hospitals over the last 10 years. This study was approved by the institutional research board. Animal subjects: All authors have confirmed that this study did not involve animal subjects or tissue. Conflicts of interest: In compliance with the ICMJE uniform disclosure form, all authors declare the following: Payment/services info: All authors have declared that no financial support was received from any organization for the submitted work. Financial relationships: All authors have declared that they have no financial relationships at present or within the previous three years with any organizations that might have an interest in the submitted work. Other relationships: All authors have declared that there are no other relationships or activities that could appear to have influenced the submitted work.

\section{Acknowledgements}

The authors would like to thank Wesley Kimble, MPH, West Virginia Clinical and Translational Science Institute.

\section{References}

1. Kastenberg ZJ, Lee HC, Profit J, Gould JB, Sylvester KG: Effect of deregionalized care on mortality in very low-birth-weight infants with necrotizing enterocolitis. JAMA Pediatr. 2015, 169:26-32. 10.1001/jamapediatrics.2014.2085

2. Backer CL, Pasquali SK, Dearani JA: Improving national outcomes in congenital heart surgery: the time has come for regionalization of care. Circulation. 2020, 141:943-945. 10.1161/CIRCULATIONAHA.119.045542

3. Chang RK, Klitzner TS: Can regionalization decrease the number of deaths for children who undergo cardiac surgery? A theoretical analysis. Pediatrics. 2002, 109:173-81. 10.1542/peds.109.2.173

4. Doyle MB, Vachharajani N, Wellen JR, et al.: A novel organ donor facility: a decade of experience with liver donors. Am J Transplant. 2014, 14:615-20. 10.1111/ajt.12607

5. Hsia R, Shen YC: Possible geographical barriers to trauma center access for vulnerable patients in the United States: an analysis of urban and rural communities. Arch Surg. 2011, 146:46-52. 10.1001/archsurg.2010.299

6. Peek-Asa C, Zwerling C, Stallones L: Acute traumatic injuries in rural populations . Am J Public Health. 2004, 94:1689-93. 10.2105/ajph.94.10.1689

7. Cunningham RM, Walton MA, Carter PM: The major causes of death in children and adolescents in the United States. N Engl J Med. 2018, 379:2468-2475. 10.1056/NEJMsr1804754

8. Osmond MH, Brennan-Barnes M, Shephard AL: A 4-year review of severe pediatric trauma in eastern Ontario: a descriptive analysis. J Trauma. 2002, 52:8-12. 10.1097/00005373-200201000-00004

9. Rutledge R, Smith CY, Azizkhank RG: A population-based multivariate analysis of the association of county demographic and medical system factors with per capita pediatric trauma death rates in North Carolina. Ann Surg. 1994, 219:205-10. 10.1097/00000658-199402000-00013

10. Dennis Vane FG, Shedd JL, Grosfeld RJ, Franiak RJ, Ulrich JC, West KW, Rescorla FJ： An analysis of pediatric trauma deaths in Indiana. J Pediatr Surg. 1990, 25:955-960. 10.1016/0022-3468(90)90237-4

11. Nicolson NG, Lank PM, Crandall ML: Emergency department alcohol and drug screening for Illinois pediatric trauma patients, 1999 to 2009. Am J Surg. 2014, 208:531-5. 10.1016/j.amjsurg.2014.06.003

12. Noffsinger DL, Wurster LA, Cooley J, et al.: Alcohol and drug screening of adolescent trauma alert patients at a level 1 pediatric trauma center. Am J Emerg Med. 2019, 37:1672-1676. 10.1016/j.ajem.2018.11.043

13. Ehrlich PF, Brown JK, Drongowski R: Characterization of the drug-positive adolescent trauma population: should we, do we, and does it make a difference if we test?. J Pediatr Surg. 2006, 41:927-30. 10.1016/j.jpedsurg.2006.01.010

14. Loiselle JM, Baker MD, Templeton JM, Schwartz G, Drott H: Substance abuse in adolescent trauma . Ann Emerg Med. 1993, 22:1530-1534. 10.1016/S0196-0644(05)81253-3

15. National Trauma Data Bank 2016. Pediatric annual report . (2016). https://www.facs.org/ /media/files/quality\%20programs/trauma/ntdb/ntdb\%20pediatric\%20annual\%20report\%202016.ash

16. UNICEF and World Health Organization: World Report on Child Injury Prevention . Peden M, Oyegbite K, Ozanne-Smith J, et al. (ed): WHO, Switzerland; 2008. 